\title{
Analysing Object-Oriented Application Frameworks Using Concept Analysis
}

\author{
Gabriela Arévalo $^{2}$ and Tom Mens ${ }^{1}$ \\ ${ }^{1}$ Postdoctoral Fellow of the Fund for Scientific Research - Flanders \\ Programming Technology Lab, Vrije Universiteit Brussel \\ Pleinlaan 2, B-1050 Brussel, Belgium \\ tom.mensevub.ac.be \\ 2 Software Composition Group, University of Berne \\ Bern, Switzerland \\ arevalo@iam.unibe.ch
}

\begin{abstract}
This paper proposes to use the formal technique of Concept Analysis to analyse how methods and classes in an object-oriented inheritance hierarchy are coupled by means of the inheritance and interfaces relationships. Especially for large and complex inheritance hierarchies, we believe that a formal analysis of how behaviour is reused can provide insight in how the hierarchy was built and the different relationships among the classes. To perform this analysis, we use behavioural information provided by the self sends and super sends made in each class of the hierarchy. The proposed technique allows us to identify weak spots in the inheritance hierarchy that may be improved, and to serve as guidelines for extending or customising an object-oriented application framework. As a first step, this paper reports on an initial experiment with the Magnitude hierarchy in the Smalltalk programming language.
\end{abstract}

\section{Introduction}

Understanding a software application implies to know how the different entities are related. In the case of an object-oriented application framework, our entities are classes and methods. When a developer defines a class in an application, he requires knowledge about how behaviour and structure have to be reused using inheritance techniques. It is not trivial to achieve optimal reuse, especially when the number of classes is large or the inheritance hierarchy is deep. In these situations, concept analysis can be used as a technique to help us cope with these problems, by analysing the inheritance and interface relationships among the classes in the class hierarchy. Then we can understand and document the way inheritance is used in the framework, and use this information to provide guidelines for how the framework can be modified or customised without running into behavioural problems or without breaching the design conventions used when building the framework.

Concept Analysis (CA) is a branch of lattice theory that allows us to identify meaningful groupings of elements (referred to as objects in CA literature) that have common properties (referred to as attributes in CA literature) ${ }^{1}$. These groupings are called con-

\footnotetext{
${ }^{1}$ We prefer to use the terms element and property instead of object and attribute because the latter terms have a specific meaning in the object-oriented paradigm.
} 
cepts and capture similarities among a set of elements based on their common properties. Mathematically, concepts are maximal collections of elements sharing common properties. They form a complete partial order, called a concept lattice, which represents the relationships between all the concepts $[1,15,6]$. To use the CA technique, one only needs to specify the properties of interest on each element, and does not need to think about all possible combination of these properties, since these groupings are made automatically by the CA algorithm.

\section{Applying concept analysis to inheritance hierarchies}

In this paper, we report on an experiment that uses concept analysis to analyse an existing inheritance hierarchy with the aim to better understand how inheritance is used in practice to achieve reuse, and to provide guidelines to improve the inheritance hierarchy. To achieve this, we analyse classes and their methods based on their relationships in terms of inheritance, interfaces and message sending behaviour. The inheritance relationship indicates whether a class is an ancestor or descendant of another one. The interface relationship indicates which methods are defined abstract or concrete in each class. The message sending behaviour indicates which methods are called by other methods in a class. Because we are mainly interested in reuse of behaviour, we will only look at self sends and super sends.

As a first step, we need to define the elements and properties we wish to reason about to apply the CA technique. Because we are interested in classes in an object-oriented hierarchy, together with their methods and the messages sent by these methods, we define an element as a pair $(C, s)$ such that "a method with signature $s$ is called (via a self send or super send) by some method implemented in the class $C$ ". For the CA properties, we chose a classification based on the relationships explained previously:

Classification based on message sending behaviour. $(C, s)$ satisfies predicate calledViaSelf if $s$ is called via a self send by some method in $C$. $(C, s)$ satisfies predicate calledViaSuper if $s$ is called via a super send by some method in $C$.

Classification based on interface relationship. $(C, s)$ satisfies predicate isConcreteIn: $D$ if $s$ is implemented as a concrete method in class $D$. $(C, s)$ satisfies predicate $i s \boldsymbol{A b}$ stractIn: $D$ if $s$ is implemented as an abstract method in class $D$.

Classification based on inheritance relationship $(C, s)$ satisfies predicate isDefinedInAncestor: $D$ if $D$ defines $s$ and is an ancestor class (i.e., a direct or indirect superclass) of $C .(C, s)$ satisfies predicate isDefinedInDescendant: $D$ if $D$ defines $s$ and is a descendant class (i.e., a direct or indirect subclass) of $C .(C, s)$ satisfies predicate isDefinedLocally if $C$ defines $s$. This means that $s$ is defined in the same class that calls it.

CA properties are then defined as conjunctions obtained by taking one predicate from each classification. Below, we present some of the properties that can be obtained by a conjunction of the predicates presented previously. 
- Predicate concreteSuperCaptureIn: $D$ is a conjunction of calledViaSuper, isConcreteIn: $D$ and isDefinedInAncestor: $D .(C, s)$ satisfies this predicate if $s$ is called via a super send in some method of $C$, and the receiver method is implemented in the class $D$ that is an ancestor class of $C$.

- Predicate concreteSelfCaptureLocally: $C$ is a conjunction of calledViaSelf, isConcreteIn: $C$ and isDefinedLocally. $(C, s)$ satisfies this predicate if $s$ is called via a self send in some method of $C$, and the receiver method is defined as a concrete one in the same class $C$.

- Predicate concreteSelfCaptureInAncestor: $D$ is a conjunction of calledViaSelf, isConcreteIn: $D$ and isDefinedInAncestor: $D .(C, s)$ satisfies this predicate if $s$ is called via a self send in some method of $C$, and the receiver method is defined as a concrete one in the class $D$ that is an ancestor class of $C$.

- Predicate concreteSelfCaptureInDescendant: $D$ is a conjunction of calledViaSelf, isConcreteIn: $D$ and isDefinedInDescendant: $D .(C, s)$ satisfies this predicate if $s$ is called via a self send in some method of $C$, and the receiver method is defined as a concrete one in the class $D$ that is a descendant class of $C$.

- Predicate abstractSelfCaptureLocally: $C$ is a conjunction of calledViaSelf, isAbstractIn: $C$ and isDefinedLocally. $(C, s)$ satisfies this predicate if $s$ is called via a self send in some method of $C$, and the receiver method is defined as an abstract one in the same class $C$.

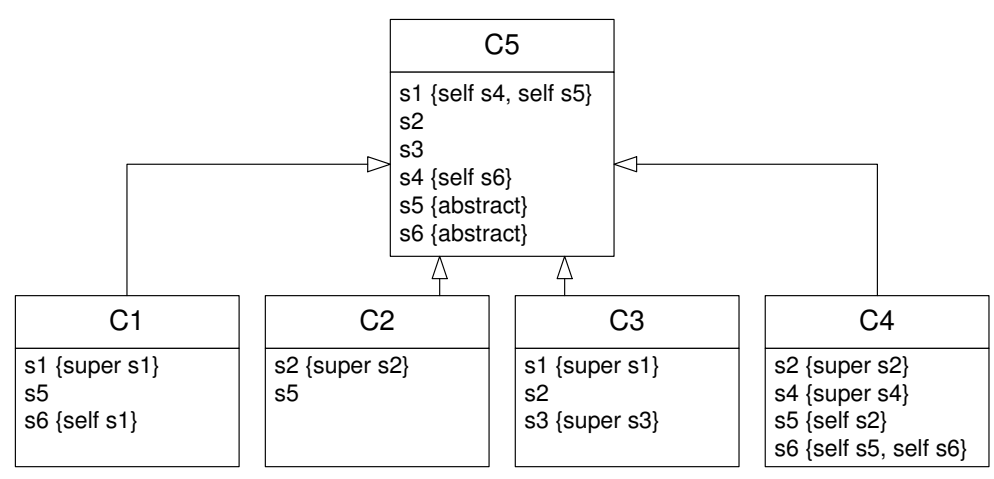

Fig. 1. Example class hierarchy

As an example of how these properties can be used to compute concepts, take a look at the example class hierarchy of Figure 1. All self sends and super sends in the source code have been annotated between curly braces. Based on this information, the CA algorithm will automatically compute the following concepts (among others):

- Concept 1 has elements $\left\{\left(C_{4}, s_{2}\right),\left(C_{4}, s_{5}\right),\left(C_{4}, s_{6}\right)\right\}$ and properties $\{$ concreteSelfCaptureLocally: $\left.C_{4}\right\}$. This means that only the selectors $s_{2}, s_{5}$ and $s_{6}$ are called via a self send that is captured by concrete method implementations in the class $C_{4}$ itself. 
- Concept 2 has elements $\left\{\left(C_{1}, s_{1}\right),\left(C_{2}, s_{2}\right),\left(C_{3}, s_{1}\right),\left(C_{3}, s_{3}\right),\left(C_{4}, s_{2}\right),\left(C_{4}, s_{4}\right)\right\}$ and properties $\left\{\right.$ concreteSuperCaptureIn: $\left.C_{5}\right\}$. This means that only the selectors $s_{1}, s_{2}, s_{3}$ and $s_{4}$ are called via a super send in the classes $C_{1}, C_{2}, C_{3}, C_{4}$ and they are implemented by a concrete method in the ancestor $C_{5}$ of these classes.

- Concept 3 has elements $\left\{\left(C_{5}, s_{5}\right),\left(C_{5}, s_{6}\right)\right\}$ and properties $\{$ abstractSelfCaptureLocally: $C_{5}$, concreteSelfCaptureInDescendant: $C_{1}$, concreteSelfCaptureInDescendant: $\left.C_{4},\right\}$. This means that abstract methods $s_{5}$ and $s_{6}$ in $C_{5}$ are defined concrete in the subclasses $C_{1}$ and $C_{4}$.

In the remainder of this paper, we will make the concept notation more compact by grouping together all selectors that belong to the same class. For example, the element set of concept 2 can be abbreviated to $\left\{\left(C_{1}, s_{1}\right),\left(C_{2}, s_{2}\right),\left(C_{3},\left\{s_{1}, s_{3}\right\}\right),\left(C_{4},\left\{s_{2}, s_{4}\right\}\right)\right\}$. We will also identify each concept by a unique number that is automatically assigned to the concept by the $\mathrm{CA}$ algorithm.

\section{Case study}

The abstract example of section 2 was only intended to make the reader understand how the process works. Our actual experiment consists of applying the CA technique to study the Magnitude inheritance hierarchy of Smalltalk in more detail ${ }^{2}$. We decided to use the Magnitude hierarchy for our first experiment because: it is sufficiently large to get meaningful results (29 classes, 894 methods); it heavily relies on code reuse by inheritance (19 abstract methods, 296 self sends, 49 super sends); it is stable and welldocumented; it is commonly available for most versions of Smalltalk. Figure 2 displays the part of the Magnitude hierarchy that is used for the examples later in this paper.

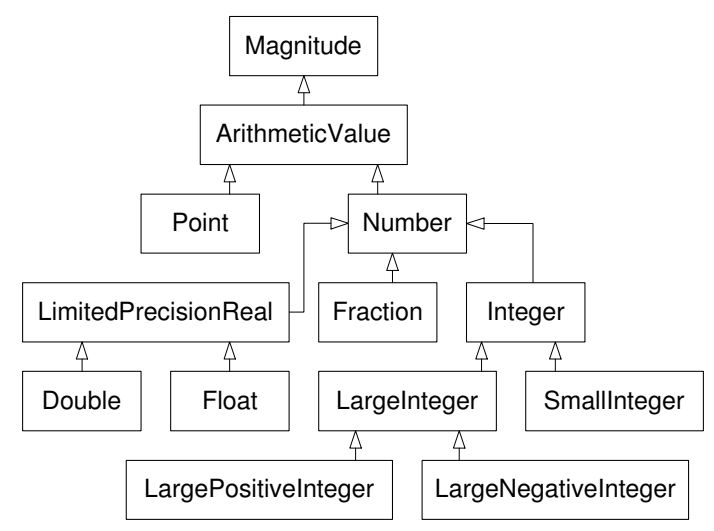

Fig. 2. Smalltalk Magnitude class hierarchy

\footnotetext{
${ }^{2}$ For our experiments, we worked in VisualWorks release 5i4, and restricted ourselves to only those classes belonging to the Smalltalk namespaces Core, Graphics, Kernel, and UI.
} 
Based on results provided by the CA algorithm, we analyzed the relationships between the classes in terms of inheritance, interface and message sending behaviour. With SOUL, a logic meta-programming language built on top of -and tightly integrated with-Smalltalk [17], we extracted 248 elements and 73 properties. ${ }^{3}$ Based on this, the CA algorithm that we implemented directly in Smalltalk computed 125 concepts as a result.

As we said previously, the properties will be of the form concreteSuperCaptureIn: $C$, concreteSelfCaptureInDescendant: $C$, abstractSelfCaptureLocally: $C$, concreteSelfCaptureLocally: $C$, concreteSelfCaptureInAncestor: $C, \ldots$ If we abstract the argument $C$ out of these properties, we find that many concepts resemble each other because they contain the same set of properties. This commonality between concepts allows us to identify concept patterns. A concept pattern consists of a textual and graphical description, a concrete example related to the Magnitude class hierarchy, and an analysis of how the pattern provides more insight in how parts of the code are reused.

\section{Concept pattern 1: Self sends captured locally}

A set of selectors $m_{1} \ldots m_{p}$ are called via a self send in a class $B$ and they are implemented in the same class. Figure 3 shows this concept pattern graphically. It occurs in 21 concepts of the Magnitude concept lattice.

For example, concept 71 has elements $\{$ (Fraction, \{reduced, negative, asFloat, asDouble $\})\}$ and properties $\{$ concreteSelfCaptureLocally: Fraction $\}$.

This concept pattern is useful to document the internal interface of a class, i.e., the set of all selectors that are implemented in the class and to which self sends are made by methods implemented in the same class. This internal interface captures and documents the core behaviour of the class. This can be used to distinguish the core methods of a class from the auxiliary ones. This is important information for reusers because, if the core methods are overridden in a subclass, all auxiliary methods will still work correctly with the new core [10].

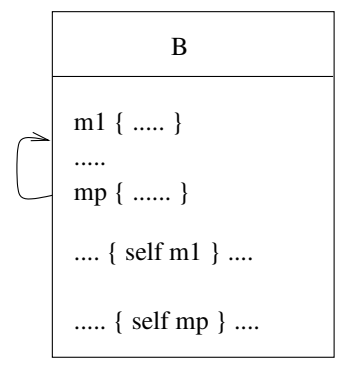

Fig. 3. Concept Pattern 1

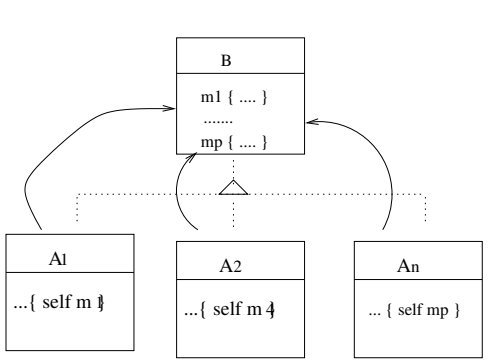

Fig. 4. Concept Pattern 2

\footnotetext{
${ }^{3}$ For our experiment we computed a static approximation of the self sends and super sends. For example, even if sends occur in a conditional branch that is never executed, they are still extracted by our algorithm.
} 


\section{Concept pattern 2: Self sends captured in ancestor}

A set of selectors $m_{1} \ldots m_{p}$ implemented in a class $B$ are called via self send in descendant classes $A_{1} \ldots A_{n}$. Figure 4 displays this concept pattern graphically. It occurs in 9 concepts of the Magnitude concept lattice.

For example, concept $\mathbf{7 3}$ has elements $\{($ LargePositiveInteger, \{digitLength, digitAt: $\}),($ LargeNegativeInteger, $\{$ digitLength, digitAt: $\})\}$ and properties \{concreteSelfCaptureInAncestor: LargeInteger\}.

This concept pattern is useful to detect the actual subclass interface of a class, i.e., the set of all selectors that are implemented in the class and to which self sends are made by subclasses. Changes to these methods will also have an impact in all subclasses that reuse it. For example, using concept $\mathbf{7 3}$ we know that if we change the implementation of digitLength or digitAt: in LargeInteger, we have to check whether the methods in LargePositiveInteger and LargeNegativeInteger that call these methods still behave as expected.

In terms of software refactoring [4], the concept pattern can sometimes be used to identify common code in sibling classes that is useful to refactor in the common superclass. For example, concept $\mathbf{7 3}$ illustrates that, to a certain extent, sibling classes LargePositiveInteger and LargeNegativeInteger reuse the behaviour defined in their superclass LargeInteger in the same way. Further investigation of the actual source code allows us to discover that the self sends (digitLength: and digitAt:) are invoked from within the implementation of the method compressed in both sibling classes and the implementation of this method is very similar in both cases. Hence, a refactoring might be appropriate to extract this common behaviour into an auxiliary method that can be pulled up into the common superclass LargeInteger. This analysis showed us a limitation of our approach: we should not only take the receiver of a self send into account (in this case digitLength and digitAt:) but also the sender (in this case compressed), since this represents essential information.

\section{Concept pattern 3: Super call}

A set of selectors $m_{1} \ldots m_{p}$ implemented in the class $B$ are called via a super send in descendant classes $A_{1} \ldots A_{n}$. Figure 5 illustrates this concept pattern graphically. It occurs in 8 concepts of the Magnitude concept lattice.

For example, concept 105 has elements $\{($ Float,$\{>, \geq, \leq\}),($ Double, $\{>, \geq, \leq\})$, (SmallInteger, $\{>, \geq, \leq\})$, (LargeInteger, $\{>, \geq, \leq\})\}$ and properties $\{$ concreteSuperCaptureIn: Magnitude\}.

This concept pattern can be used to detect the actual overriding interface of a class, i.e., the set of all selectors that are implemented in the class and to which super sends are made by methods implemented in descendants. For example, concept 105 shows that $\{>, \geq, \leq\}$ is an important part of the overriding interface of Magnitude, since each of these selectors are overridden in descendant classes Float, Double, SmallInteger and LargeInteger for optimisation purposes.

The concept pattern can also detect situations of implementation inheritance [11]. Typically, when implementation inheritance is used, a class overrides many methods defined in its parent (and uses super sends to invoke the parent behaviour). Finally, the 
concept pattern can provide guidelines for framework customisation. If we define a new subclass of a given class, it is likely that we have to override the methods specified in the overriding interface of the parent class. For example, if we would decide to create a new subclass of LimitedPrecisionReal or Integer, it is very likely that we need to override all the selectors in $\{>, \geq, \leq\}$.

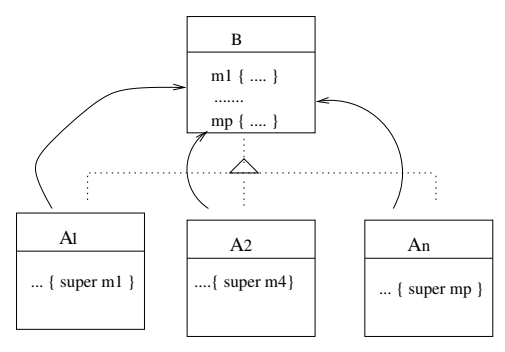

Fig. 5. Concept Pattern 3

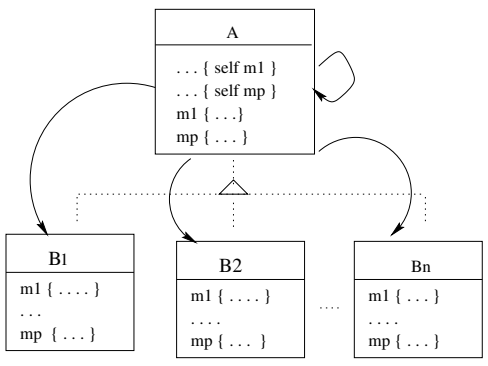

Fig. 6. Concept Pattern 4

\section{Concept pattern 4: Local self send captured in descendants}

A set of selectors $m_{1} \ldots m_{p}$ are called via a self send in the class $A$ and the selectors are implemented in $A$ and in some of its descendant classes $B_{1} \ldots B_{k}$. Figure 6 shows this concept pattern in a general way. It occurs in 31 concepts of the Magnitude concept lattice.

For example, concept 69 has elements $\{($ Number, $\{$ raisedTo:, sqrt, ln, truncated $\})\}$ and properties \{concreteSelfCaptureInDescendant: Float, concreteSelfCaptureInDescendant: Double, concreteSelfCaptureLocally: Number\}.

This concept pattern documents which specific methods are overridden in the subclasses of a common superclass. This means that the superclass defines some common or default behaviour for these methods, and each of the descendants can override this implementation via the mechanism of late binding with subclass-specific behaviour.

\section{Concept pattern 5: Local self send with super delegation}

A set of selectors $m_{1} \ldots m_{p}$ are called via a self and super send in a class $A$ and the selectors are implemented in the same class $A$ as well as in an ancestor class $B$. Figure 7 illustrates this concept pattern graphically. It occurs in 4 concepts of the Magnitude concept lattice. For example, concept 48 has elements $\{($ Smallinteger, $\{>, \geq, \leq\})\}$ and properties \{(concreteSuperCapture: Magnitude, concreteSelfCaptureLocally: Smallinteger\}

This concept pattern documents delegation between methods in the same class and with the superclass. In all the found cases, the method that calls a selector via a super send has the same name as the selector itself. For example, in SmallInteger the method 
$\geq$ contains a "super $\geq$ " statement. This means that part of the action to be executed (when a self send is made) is defined in the superclass, and the message is delegated by a super send.

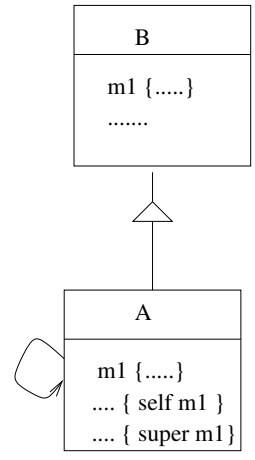

Fig. 7. C. Pattern 5

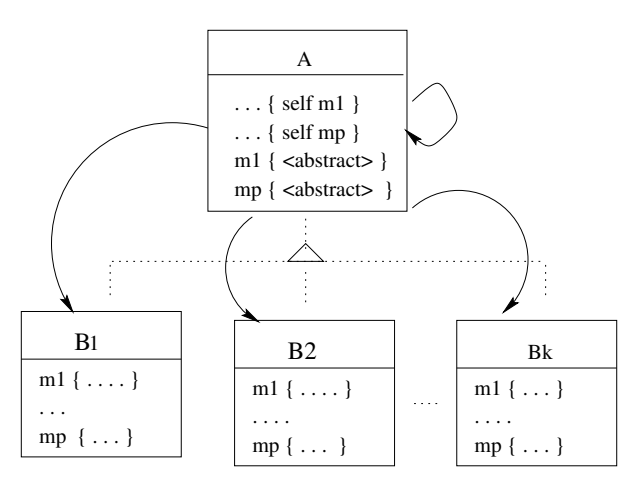

Fig. 8. Concept Pattern 6

\section{Concept pattern 6: Template methods and hook methods}

A set of selectors $m_{1} \ldots m_{p}$ are called via a self send in a class $A$ and the selectors are implemented as abstract methods in the same class $A$ and are implemented as concrete methods in descendant classes $B_{1} \ldots B_{k}$. Figure 8 illustrates this concept pattern graphically. It occurs in 7 concepts of the Magnitude concept lattice.

In the Magnitude hierarchy, this concept pattern only occurs for the subhierarchies with root classes Integer and ArithmeticValue. For example, concept 31 has elements $\{($ ArithmeticValue, $\{*,-\})\}$ and properties \{abstractSelfCaptureLocally: ArithmeticValue, concreteSelfCaptureInDescendant: $\{$ LargeInteger, Fraction, Integer, SmallInteger, Float, FixedPoint, Point, Double\} \}.

In this example, the abstract methods $\{*,-\}$ in ArithmeticValue are called by other methods of the same class, but the actual implementation is defined in descendant classes. This concept pattern identifies the hot spots in an object-oriented application framework $[9,3]$. These hot spots are implemented by means of so-called template methods and hook methods $[16,5]$. In their simplest form, template methods are methods that perform self sends to abstract methods, which are the hook methods that are expected to be overridden in subclasses.

The information expressed in this concept pattern identifies the abstract interface of a class, as well as the subclasses that provide a concrete implementation of this interface. This information is essential during framework customisation when we want to add a concrete subclass of an abstract class, because it tells us which methods should be at least be implemented. 


\section{Related work}

Godin and Mili $[8,7]$ used concept analysis to maintain, understand and detect inconsistencies in the Smalltalk Collection hierarchy. They showed how Cook's [2] earlier manual attempt to build a better interface hierarchy for this class hierarchy (based on interface conformance) could be automated. In C++ and Java, Snelting and Tip [13] analysed a class hierarchy by making the relationship between methods and variables explicit. They were able to detect design anomalies such as class members that are redundant or that can be moved into a derived class. The approach proved useful to serve as a basis for automated or interactive restructuring tools for class hierarchies. Siff and Reps [12] used concept analysis to modularise legacy $\mathrm{C}$ programs into $\mathrm{C}++$ classes. Last but not least, Tonella and Antoniol [14] used concept analysis to infer structural design patterns from $\mathrm{C}++$ code, which also provides crucial information to get a deeper understanding of object-oriented application frameworks.

All the above approaches only took information into account about which selectors are implemented by which classes. More behavioural information (e.g., based on self and super sends) was not considered. Hence, they could only detect interface inheritance but not implementation inheritance. As shown in this paper, more behavioural information about how a subclass is derived from its subclass is essential to analyse and understand the kind of reuse that is achieved.

\section{Conclusion and Future Work}

In this paper we analysed the well-known Magnitude inheritance hierarchy in Smalltalk using Concept Analysis. Based on information about self sends, super sends and invoked methods, we calculated the concept lattice for this hierarchy. We classified the generated concepts into concept patterns, which provide a roadmap of the code that ought to be analysed and understood. With the information given by the concept patterns, we discovered a number of interesting non-documented relationships about how classes and methods in the hierarchy are reused. A preliminary analysis of these patterns strengthened our belief that the technique is useful to: document the subclass interface of a class; provide guidelines on how an object-oriented framework can be customised or reused; identify hot spots in an object-oriented application framework; detect the type of inheritance (e.g. interface inheritance or implementation inheritance) used in an inheritance hierarchy; identify opportunities for refactoring; get insight in the potential impact of changes to framework classes. Based on these results, we believe that Concept Analysis is a promising technique in the understanding and re-engineering of large inheritance hierarchies.

Based on these results, we know that a lot of further research is necessary. One research avenue concerns the applicability of CA. We intend to confirm the usefulness of our method by analysing other well-known and non-trivial Smalltalk class hierarchies (e.g., Collection, Model, View and Controller). We also want to apply our approach to other object-oriented languages (such as Java and $\mathrm{C}++$ ) to investigate the effect of language-specific properties (such as interfaces or multiple inheritance) by comparing similar class hierarchies in different languages. Another topic of future work is to investigate the effect of other behavioural information such as method invocations, variable 
accesses and variable updates; or the effect of other essential relationships between classes, such as composition and aggregation. Finally, we should take into account the additional information provided by how the concepts in the generated concept lattice are related via a partial order.

\section{Acknowledgements}

We thank Dirk Deridder, Oscar Nierstrasz, Roel Wuyts and the referees for their feedback.

\section{References}

1. G. Birkhoff. Lattice theory. American Mathematical Society, 1940.

2. W. R. Cook. Interfaces and specifications for the smalltalk-80 collection classes. In Proc. Int'l Conf. Object-Oriented Programming Systems, Languages, and Applications, volume 27(10) of ACM SIGPLAN Notices, pages 1-15. ACM Press, October 1992.

3. S. Demeyer. Analysis of overriden methods to infer hot spots. In S. Demeyer and J. Bosch, editors, ECOOP '98 Workshop Reader, volume 1543 of Lecture Notes in Computer Science. Springer-Verlag, 1998.

4. M. Fowler. Refactoring: Improving the Design of Existing Programs. Addison-Wesley, 1999.

5. E. Gamma, R. Helm, R. Johnson, and J. Vlissides. Design Patterns: Elements of Reusable Object-Oriented Languages and Systems. Addisson-Wesley, 1994.

6. B. Ganter and R. Wille. Formal Concept Analysis: Mathematical Foundations. Springer Verlag, 1999.

7. R. Godin, H. Mili, G. W. Mineau, R. Missaoui, A. Arfi, and T.-T. Chau. Design of class hierarchies based on concept (galois) lattices. Theory and Application of Object Systems, 4(2):117-134, 1998.

8. R. Godin and H. Mili. Building and maintaining analysis-level class hierarchies using galois lattices. In Proc. Int'l Conf. Oject-Oriented Programs, Systems, Languages and Applications, volume 28 of ACM SIGPLAN Notices, pages 394-410. ACM Press, October 1993.

9. R. E. Johnson and B. Foote. Designing reusable classes. J. Object-Oriented Programming, 1(2):22-35, Feb. 1988.

10. J. Lamping. Typing the specialization interface. In Proc. Int'l Conf. Oject-Oriented Programs, Systems, Languages and Applications, volume 28 of ACM SIGPLAN Notices, pages 201-214. ACM Press, October 1993.

11. B. Meyer. Object-Oriented Software Construction. Prentice Hall, second edition, 1997.

12. M. Siff and T. Reps. Identifying modules via concept analysis. In Proc. Int. Conf. Software Maintenance, pages 170-179. IEEE Computer Society Press, 1997.

13. G. Snelting and F. Tip. Reengineering class hierarchies using concept analysis. In $A C M$ Trans. Programming Languages and Systems, 1998.

14. P. Tonella and G. Antoniol. Object oriented design pattern inference. In Proc. Int'l Conf. Software Maintenance, pages 230-238. IEEE Computer Society Press, 1999.

15. R. Wille. Restructuring lattice theory: An approach based on hierarchies of concepts. Ordered Sets, Ivan Rival Ed., NATO Advanced Study Institute, pages 445-470, September 1981.

16. R. Wirfs-Brock, B. Wilkerson, and L. Wiener. Designing Object-Oriented Software. Prentice Hall, 1990

17. R. Wuyts. Declarative reasoning about the structure of object-oriented systems. In Proc. Int'l Conf. TOOLS USA'98, pages 112-124. IEEE Computer Society Press, 1998. 